DOI: http://dx.doi.org/10.18203/2320-1770.ijrcog20175052

Case Report

\title{
Common tumor at a rare site
}

\section{Anuradha Murugesan*, Karthiga Prabhu}

Department of Obstetrics and Gynecology, SRM Medical College, Kattankulathur, Kanchipuram, Tamil Nadu, India

Received: 16 August 2017

Revised: 29 August 2017

Accepted: 16 September 2017

\section{*Correspondence:}

Dr. Anuradha Murugesan,

E-mail: anuthanigai@yahoo.com

Copyright: (C) the author(s), publisher and licensee Medip Academy. This is an open-access article distributed under the terms of the Creative Commons Attribution Non-Commercial License, which permits unrestricted non-commercial use, distribution, and reproduction in any medium, provided the original work is properly cited.

\begin{abstract}
Extrauterine leiomyomas are rare and are a great diagnostic challenge. Leiomyoma and fibroma are the most common mesenchymal tumours of the vagina. 51-year-old multiparous lady presented with vulvovaginal swelling. On examination, a lobulated firm vulvovaginal mass measuring $6 \times 7 \mathrm{~cm}$, occupying the right posterolateral part of labia majora extending into the lateral vaginal wall was seen. Histopathology was suggestive of Leiomyoma. Leiomyoma should be considered as one of the differential diagnosis of solid vaginal mass.
\end{abstract}

Keywords: Fibroid, Leiomyoma, Vagina

\section{INTRODUCTION}

Leiomyoma is the most common gynaecological tumour which commonly arises from uterus and cervix. Extrauterine leiomyomas are rare and are a great diagnostic challenge. Vaginal Leiomyomas are reported to be the most common mesenchymal tumours affecting the vagina., ${ }^{1,6}$ Leiomyomas of vagina are typically painless, solitary, and are observed to be wellcircumscribed. They are generally found in middle-aged women may be influenced by hormonal factors. Vaginal Leiomyomas may cause abdominal pain, urination difficulties, and abnormal vaginal bleeding. Some tumours are known to develop in women after hysterectomy. The treatment of choice is a surgical removal of the entire tumor. The prognosis is excellent with appropriate treatment, since Leiomyoma of Vagina is a benign tumor. Here we are reporting a case of vagina leiomyoma.

\section{CASE REPORT}

Mrs X, a 51-year-old multiparous lady presented to our outpatient clinic with complaints of swelling in the external genitalia for 5 years. She had pain over the swelling for the past 5 months. She did not have any menstrual abnormalities. Her bowel and bladder habits were normal. On examination, a lobulated firm vulvovaginal mass measuring $6 \times 7 \mathrm{~cm}$, occupying the right posterolateral part of labia majora extending into the lateral vaginal wall was seen (Figure 1).

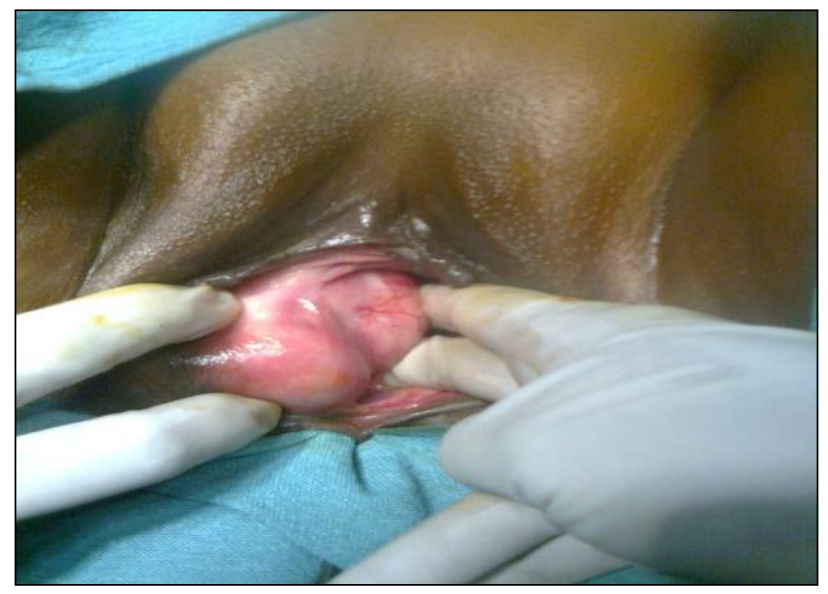

Figure 1: Vulvovaginal mass. 
Mucosa and skin over the mass was normal. Cervix was healthy and uterus was found to be normal size. Differential diagnosis of leiomyoma, fibroma was made and planned for enucleation of the mass. Ultrasonography of the pelvis was found to be normal. Under spinal anesthesia, enucleation of the mass was done. Cut section of the mass was solid with whorled appearance with no evidence of degeneration and hemorrhage. This macroscopic appearance which was suggestive of leiomyoma was confirmed by histopathology (Figure 2). Her postoperative period was uneventful and she is on follow-up for recurrence.

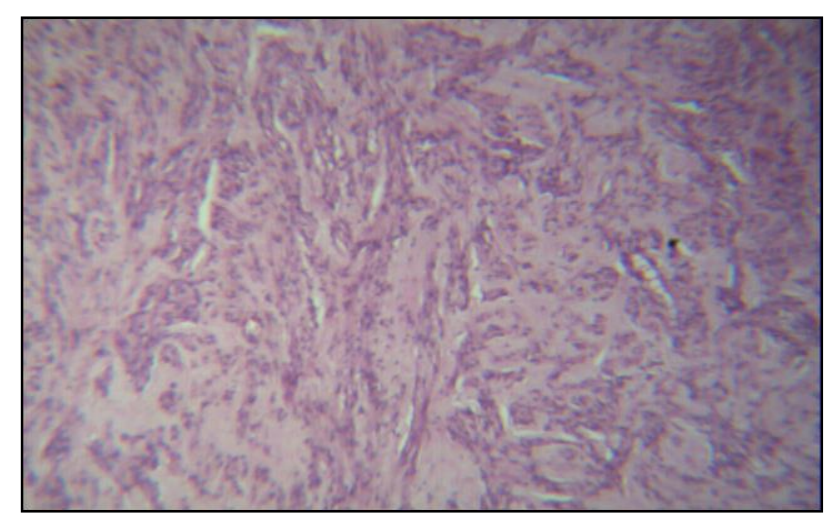

Figure 2: Histopathology suggestive of leiomyoma.

\section{DISCUSSION}

Leiomyoma usually arise from the body of the uterus and sometimes from the cervix. It can also arise from extrauterine sites like round ligament, broad ligament, uterosacral ligament, inguinal canal, ovary and very rarely from vulva and vagina. ${ }^{1}$ Primary vaginal tumors are rare. Benign solid tumors arising from vagina include Papilloma, hemangioma, mucous polyp and leiomyoma and fibroma. Leiomyoma and fibroma are the most common mesenchymal tumors of the vagina., Leiomyoma vagina is a very rare condition. Approximately 300 cases reported worldwide. ${ }^{1,3}$ First case was reported in 1733 by Denys de Leyden. It is a hormone sensitive tumor. It usually regresses after menopause. Leiomyoma vagina recurrent in pregnancy has been reported strongly indicating hormone dependency. ${ }^{2}$ Most of the lesions are asymptomatic. Symptoms are mainly due to compression causing dysuria, frequency and urinary retention, dyspareunia and pelvic pain. It usually presents as a bulging vaginal mass and presents as a single solitary nodule occurring in the age group 35 to 50 years from the anterior vaginal wall. ${ }^{1}$ But in our patient leiomyoma was in the posterolateral wall. A case of paraurethral leiomyoma also reported. ${ }^{4}$ Macroscopic appearance is firm well circumscribed resembling uterine fibroids with cut section showing whorled appearance. Microscopic appearance shows fascicles of uniform smooth muscle with typical spindle configuration and abundant pale eosinophilic cytoplasm. They may be confused with cervical fibroid, other benign and malignant neoplasm of vagina, bartholins cyst, gartners cyst, cervical polyp and cystocele. ${ }^{5}$ Treatment is always surgical. Excision or enucleation is done by vaginal route, abdominal route or both abdomino-vaginal approach depending upon the site. ${ }^{3}$ Surgical removal should always be done to prevent growth and sarcomatous change. ${ }^{7}$ Local recurrences have been reported after excision. In case of recurrences ovariectomy should be done as it a hormone sensitive tumor. ${ }^{8}$ Rapid growth and tumor recurrences usually indicate malignancy. ${ }^{7}$ Sarcomatous change have been reported.

\section{CONCLUSION}

Leiomyoma should be considered as one of the differential diagnosis of solid vaginal mass.

Funding: No funding sources

Conflict of interest: None declared

Ethical approval: Not required

\section{REFERENCES}

1. Park SJ, Choi SJ, Han KH, Park KH, Chung H, Song JM. Leiomyoma of the vagina that caused cyclic retention. Acta Obstetricia et Gynecologica. 2007;86:102-10

2. Rywlin AM, Simmons RJ, Robinson MJ. Leiomyoma of vagina recurrent in pregnancy: a case with apparent hormone dependency. South Med J. 1969;62(12):1449-51.

3. Gowri R, Soundararaghvan S, Oumachigui A, Sistla $\mathrm{SC}$, Iyengar KR. Leiomyoma of the vagina: an unusual presentation. J Obstet Gynaecol Res. 2003;29(6):395-8.

4. Coumary SA, Ghose S, Samal R. Paraurethral leiomyoma in a woman: a Case report. Int J Reprod Contracept Obstetet Gynecol. 2013;2(1):99-100.

5. Agarwal S, Yadav R, Sangwan K, Dahiya P. Vaginal Leiomyoma: a rare case of menometrorrhagia. Int $\mathbf{J}$ Radiol. 2007;9(1).

6. Kumar M, Sadaf L, Raghuvanshi S, Patne S. Epithelioid leiomyoma of vagina. Internet J Gynecol Obstet. 2009;12(2).

7. Horn LC, Fischer U, Reuter S, Pytlel C. Leiomyosarcoma and Leiomyoma of vagina. Zentralbl gynakol 1998:120(1):38-41.

8. Dhaliwal LK, Das I, Gopalan S. Recurrent Leiomyoma of Vagina. Int $\mathbf{J}$ Gynecol Obstet 1992;37(4):281-3.

Cite this article as: Murugesan A, Prabhu K. Common tumor at a rare site. Int J Reprod Contracept Obstet Gynecol. 2017;6:5181-2. 